\title{
HANDBOOK OF CARBON NANO MATERIALS
}

Volume 3

Medicinal and Bio-related Applications 


\section{HANDBOOK OF CARBON NANO MATERIALS}

Volume 4

Materials and Fundamental Applications 
This page intentionally left blank 


\title{
HANDBOOK OF \\ CARBON NANO MATERIALS
}

\author{
Volume 3 \\ Medicinal and Bio-related Applications
}

\section{Editors \\ Francis D'Souza \\ University of North Texas, USA \\ Karl M. Kadish}

University of Houston, USA 


\title{
HANDBOOK OF \\ CARBON NANO MATERIALS
}

\author{
Volume 4 \\ Materials and Fundamental Applications
}

\section{Editors \\ Francis D'Souza \\ University of North Texas, USA \\ Karl M. Kadish}

University of Houston, USA 


\section{Published by}

World Scientific Publishing Co. Pte. Ltd.

5 Toh Tuck Link, Singapore 596224

USA office: 27 Warren Street, Suite 401-402, Hackensack, NJ 07601

UK office: 57 Shelton Street, Covent Garden, London WC2H 9HE

\section{British Library Cataloguing-in-Publication Data}

A catalogue record for this book is available from the British Library.

\section{HANDBOOK OF CARBON NANO MATERIALS \\ (In 2 Volumes) \\ Volume 3: Medicinal and Bio-related Applications}

Copyright (C) 2012 by World Scientific Publishing Co. Pte. Ltd.

All rights reserved. This book, or parts thereof, may not be reproduced in any form or by any means, electronic or mechanical, including photocopying, recording or any information storage and retrieval system now known or to be invented, without written permission from the Publisher.

For photocopying of material in this volume, please pay a copying fee through the Copyright Clearance Center, Inc., 222 Rosewood Drive, Danvers, MA 01923, USA. In this case permission to photocopy is not required from the publisher.

ISBN-13 978-981-4401-41-8 (Set)

ISBN-10 981-4401-41-2 (Set)

ISBN-13 978-981-4401-42-5 (Vol. 3)

ISBN-10 981-4401-42-0 (Vol. 3)

Typeset by Stallion Press

Email: enquiries@stallionpress.com

Printed in Singapore. 


\section{Published by}

World Scientific Publishing Co. Pte. Ltd.

5 Toh Tuck Link, Singapore 596224

USA office: 27 Warren Street, Suite 401-402, Hackensack, NJ 07601

UK office: 57 Shelton Street, Covent Garden, London WC2H 9HE

\section{British Library Cataloguing-in-Publication Data}

A catalogue record for this book is available from the British Library.

\section{HANDBOOK OF CARBON NANO MATERIALS \\ (In 2 Volumes) \\ Volume 4: Materials and Fundamental Applications}

Copyright (C) 2012 by World Scientific Publishing Co. Pte. Ltd.

All rights reserved. This book, or parts thereof, may not be reproduced in any form or by any means, electronic or mechanical, including photocopying, recording or any information storage and retrieval system now known or to be invented, without written permission from the Publisher.

For photocopying of material in this volume, please pay a copying fee through the Copyright Clearance Center, Inc., 222 Rosewood Drive, Danvers, MA 01923, USA. In this case permission to photocopy is not required from the publisher.

ISBN-13 978-981-4401-41-8 (Set)

ISBN-10 981-4401-41-2 (Set)

ISBN-13 978-981-4401-43-2 (Vol. 4)

ISBN-10 981-4401-43-9 (Vol. 4)

Typeset by Stallion Press

Email: enquiries@stallionpress.com

Printed in Singapore. 


\section{Contents}

Preface $\quad$ xi

List of Contributors $\quad$ xiii

Contents of Volumes 3 and $4 \quad$ xix

$1 /$ Fullerenes in Biology and Medicine 1

Marco Carini, Luka Dordević and Tatiana Da Ros

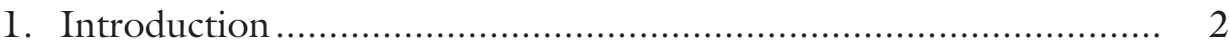

2. Fullerene Cluster Formation .................................................... 2

3. Fullerene Toxicity ............................................................... 3

4. Cellular Uptake and Membrane Interactions ................................ 6

5. $\mathrm{C}_{60}$ Derivatives as Neuroprotective Agents ................................. 9

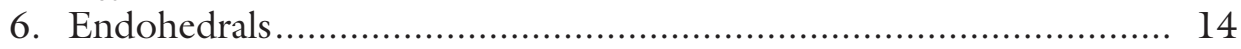

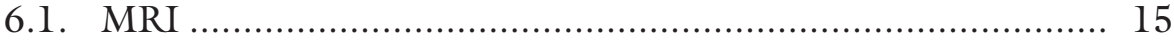

6.2. Radionuclides ............................................................... 17

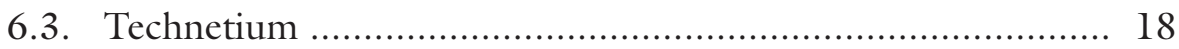

7. Photocleavage and Photodynamic Therapy ................................ 18

7.1. DNA photocleavage ...................................................... 19

7.2. Anticancer photodynamic therapy ….................................. 21

8. Inhibition of Enzymes, Viruses and Bacteria ............................. 25

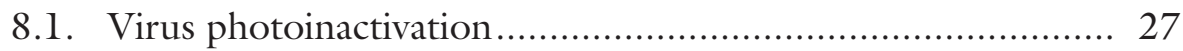

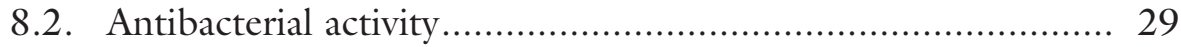

9. Drug and Gene Delivery ....................................................... 30

10. Miscellaneous Applications of Fullerene .................................. 37

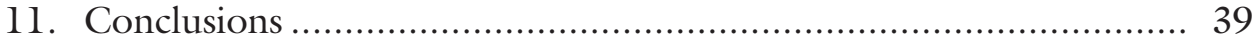

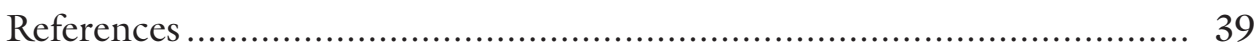

2 / Toxicity Studies of [60]Fullerene and Carbon Nanotubes: 49 State of the Art

Jelena Kolosnjaj-Tabi, Tarek Baati, Henvi Szwarc and Fatbi Moussa

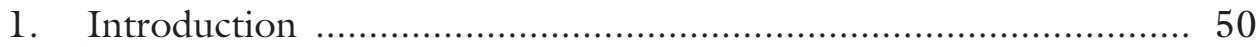

2. Toxicity Studies of [60]Fullerene …..................................... 50 
2.1. General considerations …............................................ 50

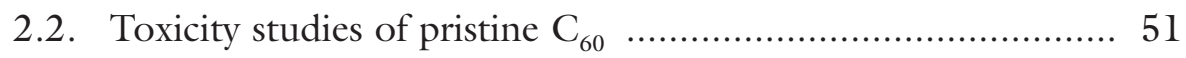

2.3. Toxicity studies of $\mathrm{C}_{60}$ derivatives ................................... 59

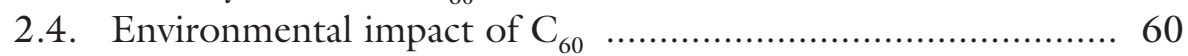

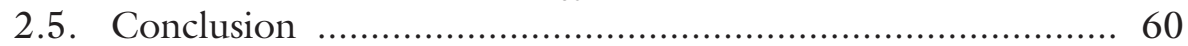

3. Toxicity Studies of Carbon Nanotubes .......................................... 61

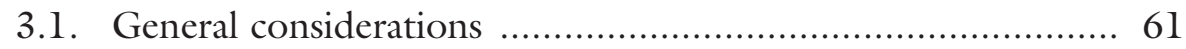

3.2. In vitro studies of CNT toxicity ....................................... 62

3.3. Toxicity studies related to the exposure at work place .......... 62

3.4. Toxicity studies related to the asbestos fibres analogy ........... 65

3.5. Toxicity studies after enteral and parenteral administrations ..... 67

3.6. Toxicity studies after oral administration ............................. 74

3.7. Conclusion ................................................................. 75

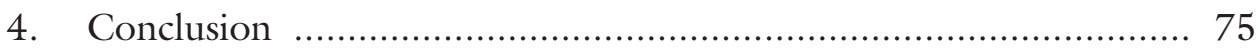

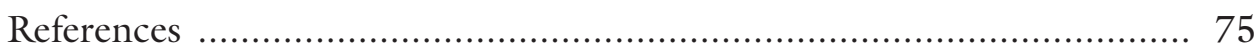

\section{3 / Electrophoretic Methods to Quantify Carbon} Nanotubes in Biological Cells

Paul Pantano, Rockford K. Draper, Carole Mikoryak and Rubung Wang

1. Introduction and Problem Statement ............................................ 84

2. Methods to Detect Carbon Nanotubes in Cells............................ 85

2.1 Optical and electron microscopies ..................................... 86

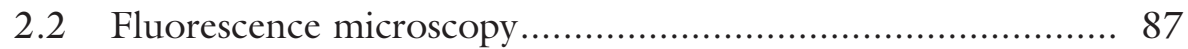

2.3 Raman microscopy ...................................................... 88

2.4 Photoluminescence microscopy .......................................... 90

2.5 Comparison to methods to quantify metal nanoparticles in cells ......................................................... 91

3. The Use of Electrophoresis in Applications Involving Carbon

Nanotubes ......................................................................... 91

3.1 Electrophoretic techniques for characterizing carbon nanotube/ligand interactions .......................................... 92

3.2 Electrophoretic techniques for carbon nanotube

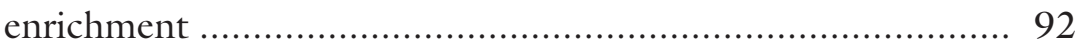

4. Electrophoretic Methods to Quantify Carbon Nanotubes in Liquid Samples ............................................................. 93

4.1 Capillary electrophoresis of dispersions of dye-labeled carbon nanotubes ........................................................... 93

4.2 Capillary electrophoresis of cells exposed to dye-labeled carbon nanotubes 
4.3 Gel electrophoresis of dispersions of carbon nanotubes ....... 95

4.4 Gel electrophoresis of cells exposed to carbon nanotubes..... 98 $5 \quad$ Conclusions and Perspectives ................................................. 100

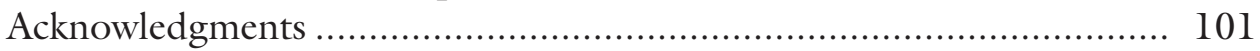

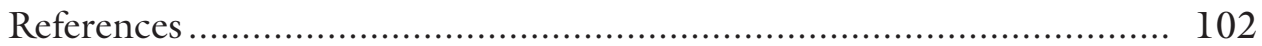

4 / Carbon Nanotechnology in Regenerative Medicine 107 Gaurav Lalwani and Balaji Sitharaman

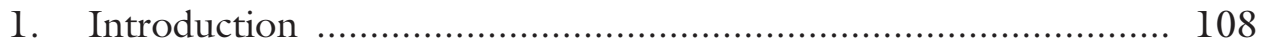

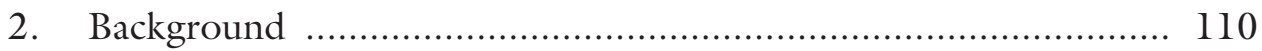

3. Bioimaging in Regenerative Medicine ................................... 116

3.1. MRI contrast agents ............................................... 117

3.2. X-ray imaging contrast agents ..................................... 120

4. Bone Tissue Engineering ....................................................... 121

5. Antioxidants and Neuroprotective Agents ............................. 122

6. Photodynamic Therapy in Regenerative Medicine .................... 126

7. Drug and Gene Delivery .................................................. 129

8. Radiotracers and Radiopharmaceuticals ................................... 134

9. In Vitro and In Vivo Toxicity Studies ..................................... 135

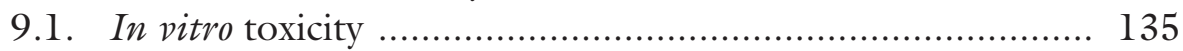

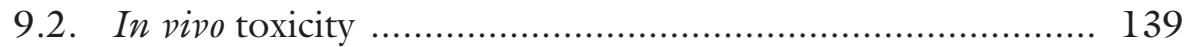

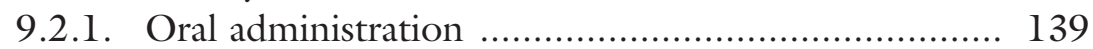

9.2.2. Inhalation (pulmonary administration) .................. 140

9.2.3. Dermal exposure .......................................... 142

9.2.4. Intraperitoneal administration ............................. 142

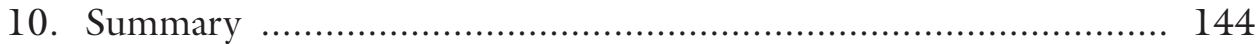

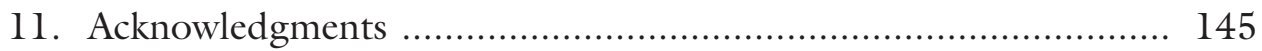

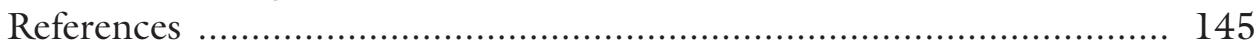

5 / Carbon Nanotube-Based Chemo- and Biosensors 151 Piyush Sindhu Sharma, Francis D'Souza and Wlodzimierz Kutner

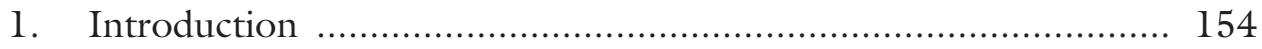

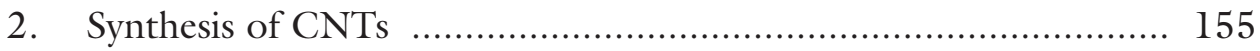

3. Purification of CNTs ........................................................ 156

4. Application of CNTs in Chemosensing ................................... 158

4.1. Chemosensing of drugs …...................................... 160

4.1.1. Chemosensing of antibiotics .............................. 160

4.1.2. Chemosensing of analgesics .............................. 164

4.1.3. Chemosensing of neural and hypertension treatment drugs 
4.1.4. Chemosensing of anticancer drugs ...................... 170

4.1.5. Chemosensing of other drugs ............................ 173

4.2. Chemosensing of pesticides ........................................ 178

4.3. Chemosensing of warfare agents and explosives ................ 188

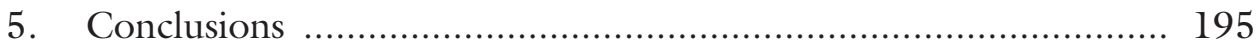

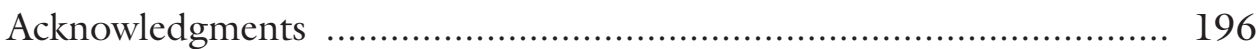

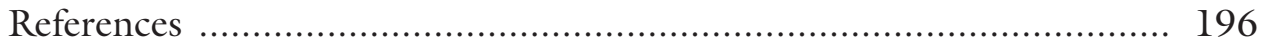

6 / Separation of Left- and Right-Handed Structures of 203 Single-Walled Carbon Nanotubes through Molecular Recognition

Gang Liu, Feng Wang, Xiaobin Peng, A. F. M. Mustafizur Rabman, Ajoy Kumar Bauri and Naoki Komatsu

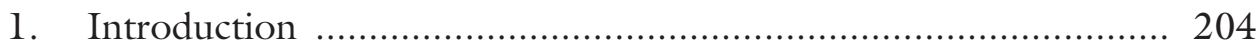

2. Terminology of SWNTs _................................................ 204

3. Recent Progress in Separation of SWNTs ............................... 207

4. Our Strategy for SWNT Separation ...................................... 213

4.1. Synthesis and structures of chiral diporphyrin nanotweezers ... 214

4.2. Extraction of SWNTs with chiral diporphyrin nanotweezers ... 216

4.3. $(n, m)$ Enrichment ...................................................... 219

4.4. Optical enrichment ..................................................... 222

5. Concluding Remarks ............................................................ 225

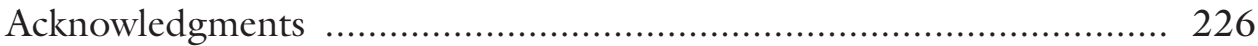

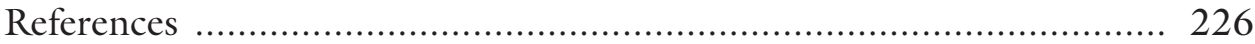

7 / Surgery at the Molecular Level: Synthesis and 233

Modifications of Open-Cage Fullerene Derivatives

Manolis M. Roubelakis and Georgios C. Vougioukalakis

1. Introduction ............................................................... 234

2. Methods to Create and Enlarge an Opening on

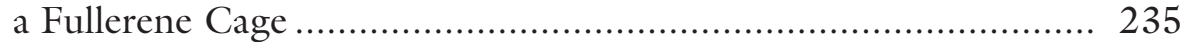

2.1. Preparation of fulleroids and azafulleroids ........................ 235

2.2. Intramolecular $[4+4]$ cycloadditions followed by

$[2+2+2]$ cycloreversions......................................... 243

2.3. Self-photooxygenation and oxidation reactions .................. 251

2.4. Nucleophilic additions on the rim of the orifice ................. 260

2.4.1. Ring expansions with hydrazines, hydrazones, and 1,2-phenylenediamines ................................. 260

2.4.2. Sulfur and selenium atom insertions..................... 265 
2.4.3. Sodium borohydride, Grignard reagents, and $\mathrm{N}$-methylmorpholine $\mathrm{N}$-oxide additions................ 269

2.5. Starting from fullerene-mixed peroxides.......................... 270

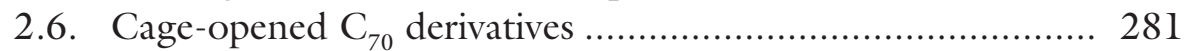

3. Aperture-Closing Methods..................................................... 283

4. Conclusions and Perspectives ..................................................... 287

Acknowledgments ............................................................ 288

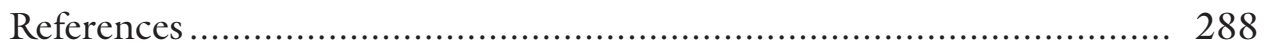

Cumulative Index of Volumes 3 and 4 


\section{Contents}

Preface $\quad$ xi

List of Contributors $\quad$ xiii

Contents of Volumes 3 and $4 \quad$ xix

1 / Functionalization of Graphene 1

Nikolaos Karousis, Solon Economopoulos and Nikos Tagmatarchis

1. Introduction............................................................................... 1

2. Graphene Production ......................................................... 2

3. Graphene Oxide .................................................................... 3

4. Reduced Graphene Oxide..................................................... 5

5. Functionalization of Reduced Graphene Oxide ........................... 12

6. Functionalization of Graphene Oxide ........................................ 20

7. Covalent Functionalization of Graphene ...................................... 28

8. Non-Covalent Functionalization of Graphene................................. 34

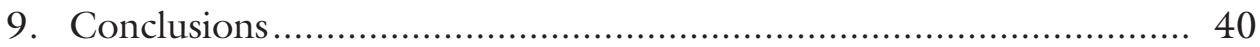

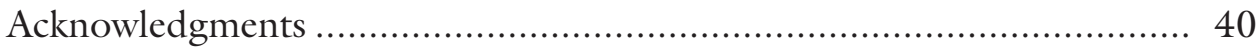

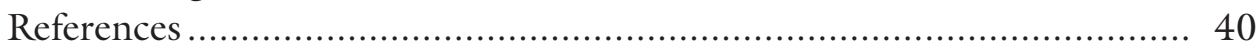

2 / Advanced Carbon Materials for Electrochemical Energy Conversion and Storage $\quad 55$

Junbo Hou, Jiajun Wang, Xueliang Sun and Michael W. Ellis

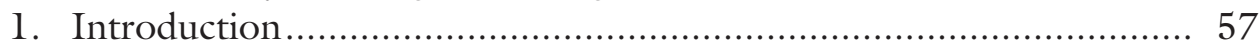

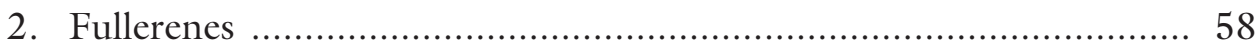

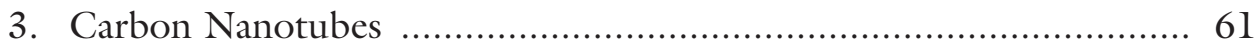

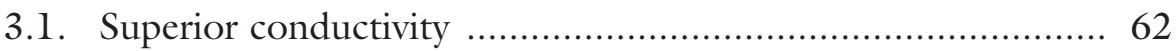

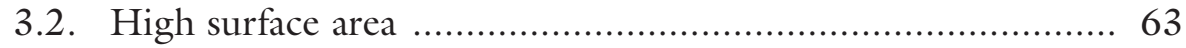

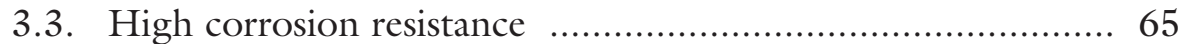

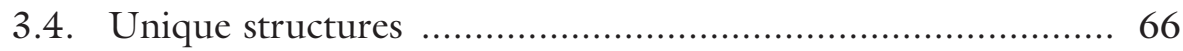

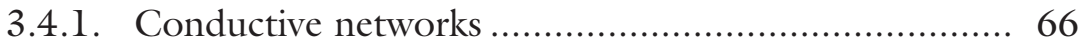

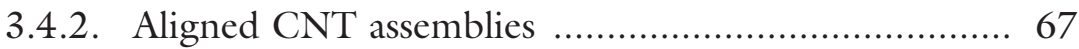

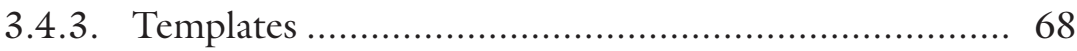


3.5. Volume buffering for lithium-ion batteries application ........ $\quad 70$

3.6. Lithium ion insertion.................................................. $\quad 72$

3.7. Foreign atom doping ................................................... 73

3.7.1. Fuel cell .......................................................... 73

3.7.2. Lithium-ion batteries ........................................ 74

3.7.3. Lithium-oxygen batteries ...................................... 75

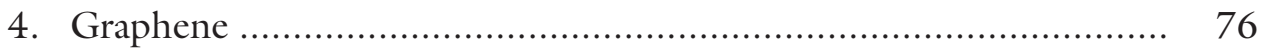

4.1. High conductivity ................................................. 76

4.2. Enhanced surface area ................................................... 77

4.3. Enhanced durability .................................................... 81

4.4. Graphene structures................................................. 81

4.5. Graphene composites................................................ 84

4.5.1. Graphene-conducting polymer hybrids ................. 84

4.5.2. Graphene-metal oxide composites ....................... 86

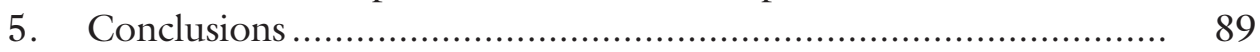

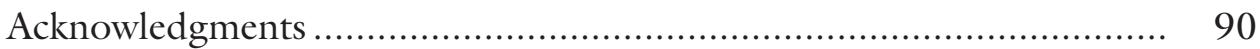

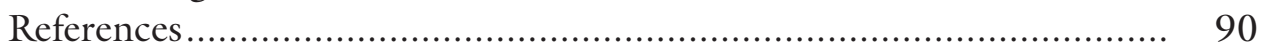

3 / Carbon Nanomaterial-Based Molecular Architectures for Light Energy Conversion $\quad 95$ Taku Hasobe

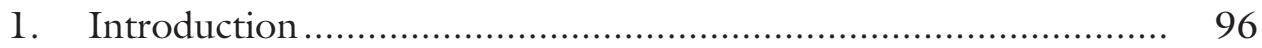

2. Fullerene-Based Molecular Composites ...................................... 98

2.1. Molecular composites of fullerenes and porphyrins ............ 98

2.2. Fullerene-porphyrin dyad for charge separation ................. 100

2.3. High-order organization between fullerene and porphyrin units using gold nanoparticle .......................... 102

2.4. Dendritic structure .................................................. 106

2.5. Porphyrin-peptide oligomers ....................................... 108

2.6. Photocurrent generation mechanism in supramolecular composites between fullerenes and porphyrins .................. 112

3. CNT-Based Molecular Composites ......................................... 114

3.1. SWCNT-driven aggregation of protonated porphyrins ........ 114

3.2. Stacked-cup carbon nanotubes: New carbon materials for light energy conversion.............................................. 116

3.3. Carbon nanohorn-porphyrin composites ......................... 119

3.4. Multi-walled carbon nanotubes-CdS nanoparticles hybrids..... 122

3.5. Graphene-porphyrin composites .................................. 123 
3.6. Polycyclic aromatic hydrocarbon-based porphyrin

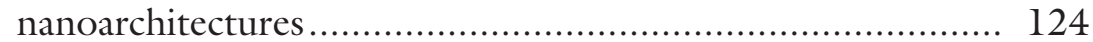

4. Conclusion and Perspectives ................................................ 125

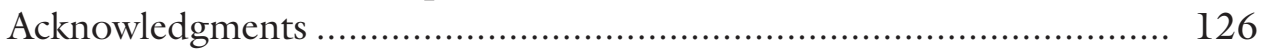

References .................................................................. 127

4 / Electrochemistry of Carbon Nanostructures: From

Pristine Materials to Functional Devices

Matteo Iurlo, Stefania Rapino, Giovanni Valenti,

Massimo Marcaccio and Francesco Paolucci

1. Introduction .......................................................................... 133

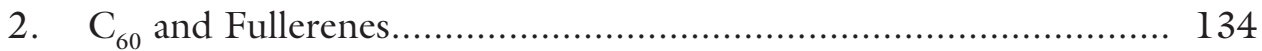

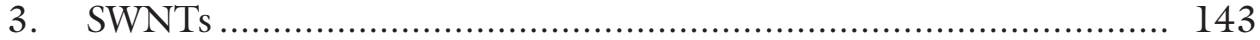

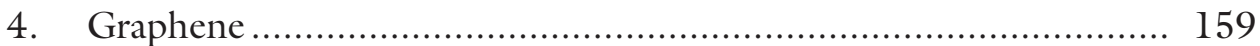

5. Comparative Electrochemistry of CNSs .................................. 163

6. CNS-Based Molecular Devices: Relevance of Their

Redox Properties ............................................................. 164

6.1. Interlocked architectures equipped with $\mathrm{C}_{60} \ldots \ldots \ldots \ldots \ldots \ldots \ldots \ldots \ldots$

7. Carbon Nanostructures in Electrochemical Devices.................... 173

7.1. Organic and dye-sensitized solar cells ............................. 175

7.2. Sensors and Biosensors ................................................... 176

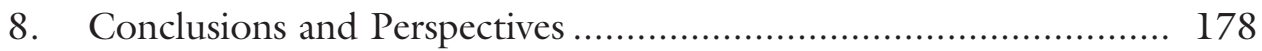

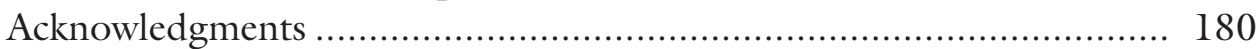

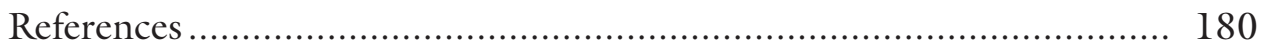

5 / Fullerene-Based Self-Assembled Morphological Materials 189 Yanfei Shen, Junko Aimi and Takashi Nakanishi

1. Introduction ................................................................. 189

2. Self-Assembled Crystalline Fullerenes with Unique Morphologies..... 190

2.1. Morphology control of fullerenes....................................... 190

2.2. Supramolecular composites ........................................ 195

3. Liquid Crystalline Assemblies ................................................ 200

4. Hierarchically Structured Organogels ....................................... 203

5. Fullerene-Based Polymers ................................................. 207

6. Conclusions and Perspectives .................................................... 211

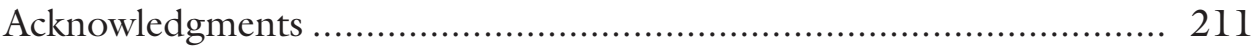

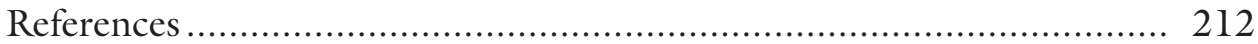


6 / Hybrids Involving Fullerenes and Carbon Nanotubes María Vizuete, Myriam Barrejón, María José Gómez-Escalonilla and Fernando Langa

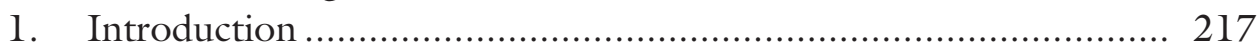

2. Encapsulated Fullerenes at CNTs ......................................... 218

3. Exohedral Fullerene-CNTs Hybrids........................................ 224

3.1. Non-covalent fullerene-CNTs hybrids .............................. 225

3.2. Covalent exohedral fullerene-CNTs hybrids...................... 232

4. Conclusions and Perspectives ...................................................... 239

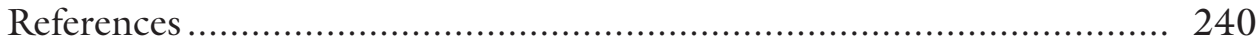

7 / Semiconductor Carbon Nanotube Optics 245

Lisa J. Nogaj, Libai Huang and Todd D. Krauss

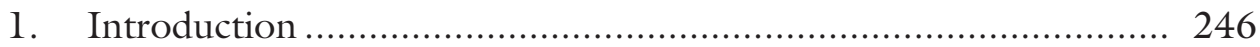

2. Nanotube Structural Properties.................................................. 248

2.1. Graphene band structure ............................................... 251

2.2. SWNT band structure............................................... 253

2.3. SWNT density of electronic states and optical transitions ..... 254

3. Excitons in Carbon Nanotubes ................................................... 257

4. Rayleigh and Raman Spectroscopy .......................................... 261

5. Absorbance and Photoluminescence........................................... 264

6. Single-Molecule Spectroscopy .................................................. 268

7. Excited State Dynamics ............................................................ 271

7.1. Exciton population dynamics of the $E_{11}{ }^{s}$ state ................. 272

7.2. Auger recombination and MEG.................................. 275

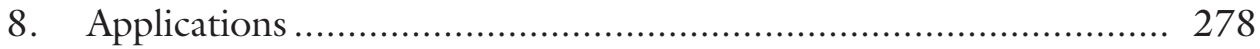

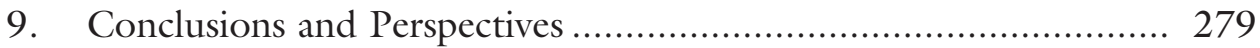

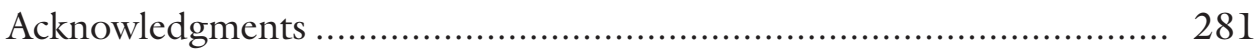

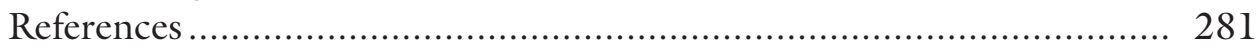

8 / Near-Field Optics of SWNTs and FRET in their 287 Nanoscale Complexes

Tetyana Ignatova, Andrei M. Nemilentsau and Slava V. Rotkin

1. Introduction ....................................................................... 288

2. Near-Field Modes of an SWNT and Spontaneous Decay

of an Emitter Coupled To It .....

2.1. Plasmonic quenching of PL: Weak emitter-field coupling regime.

2.1.1. PL quenching by a single SWNT in the free space:

Near field of plasmon-polaritons 
2.1.2. SWNT arrays in the free space: Composite plasmon-polaritons.

2.1.3. Single SWNT in a homogeneous dielectric:

Mean-field screening of the plasmon-polaritons ...... 300

2.2. Strong atom-field coupling regime: Rabi oscillations

3. Near-Field Energy Transfer Between SWNTs and

Rare Earth Ions: Spatial Correlations in Solution ....................... 305

3.1. Fundamentals of the energy transfer process ..................... 305

3.2. Time-resolved spectroscopy ....................................... 307

3.3. The shortening of $\mathrm{Tb}$ and Eu emission in the presence of an SWNT .................................................. 308

3.4. FRET-mediated acceptor emission ................................... 309

3.5. Spatial correlation analysis: FRET distance in the nanoscale complexes ............................................... 310

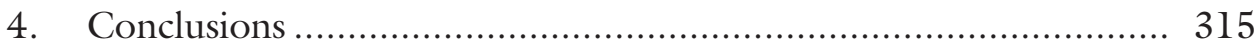

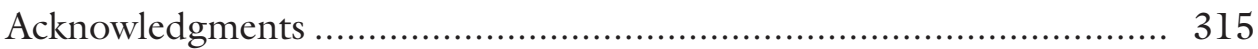

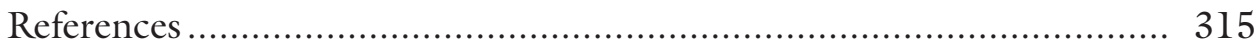

9 / Understanding the Role of the Metal Catalyst in Carbon Nanotube Growth: Towards Chiral-Selective Growth of Single-Walled Carbon Nanotubes

Wei-Hung Chiang and R. Moban Sankaran

1. Introduction ..................................................................... 322

2. Microplasma Synthesis of Metal and Alloyed Catalysts ................ 324

3. Two-Step, Floating Catalyst Process for Carbon Nanotube Growth .................................................................... 336

4. Kinetic Studies of Carbon Nanotube Growth via Aerosol Measurements.................................................................... 340

5. Diameter-Controlled Growth of Carbon Nanotubes in the Gas Phase ..................................................................... 350

6. Linking Chirality Distributions of SWCNTs to Catalyst Composition.......................................................... 357

7. Perspective ......................................................................... 374

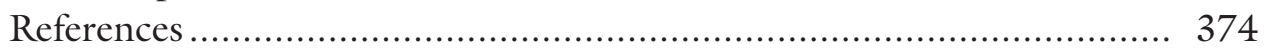

10 / Stability Computations for Fullerenes and 381 Metallofullerenes

Zdeněk Slanina, Filip Ublik, Shyi-Long Lee, Takeshi Akasaka and Shigeru Nagase

1 Introduction

2 Energetics and Thermodynamics of Carbon Clusters 
3 Stabilities of Empty Fullerenes …............................................ 387

$4 \quad$ Stabilities of Metallofullerenes................................................ 390

5 Stabilities of Non-Metal Endohedrals ........................................ 402

6 Kinetic Control...................................................................... 403

7 Stabilities of Metal/Non-Metal Endohedrals.............................. 406

8 Stabilities of Solvent-Induced Metallofullerene Derivatives .......... 410

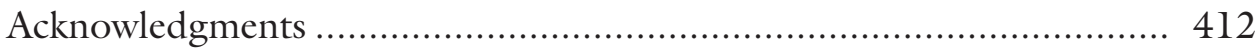

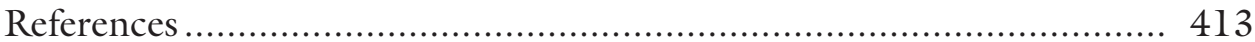

Cumulative Index of Volumes 3 and 4 
This page intentionally left blank 


\section{Preface}

We are pleased to present the second two-volume set of The Handbook of Carbon Nanomaterials. The first two-volume set was published in recognition of the $25^{\text {th }}$ anniversary of the discovery of Fullerenes, which was followed in later years by the discovery of carbon nanotubes and then graphene. These nanomaterials have taken a solid place in modern science and technology, as demonstrated by the awarding of two Nobel Prizes - the first, in Chemistry, was given in 1996 to Robert F. Curl Jr., Sir Harold Kroto and Richard E. Smalley for the discovery of fullerenes. The second, in Physics, was awarded in 2010 to Andre Geim and Konstantin Novoselov for the isolation of graphene. Since 1985, more than 110,000 scientific papers have been published on topics devoted to fullerenes, carbon nanotubes and related materials and there is no doubt that these types of carbon nanomaterials, with their unique structures and properties, will continue to find many new applications in all areas of science, medicine and technology.

The current Handbook of Carbon Nanomaterials consists of 17 chapters in two volumes; the first volume compiles chapters from leading authors on medicinal and bio-related applications while the second volume covers topics ranging from materials to fundamental applications. Specific topics covered in Volume 3 include fullerenes in biology and medicine, the current state of the art of toxicity studies of fullerenes and carbon nanotubes, methods of detection of carbon nanotubes in living cells, carbon nanotechnology in regenerative medicine, chemical and biochemical sensors based on carbon nanotubes, molecular recognition approach for separating left- and right-handed structures of single wall carbon nanotubes and open cage fullerenes. The specific topics covered in Volume 4 are functionalization of graphene, electrochemical conversion and storage of energy, nanocarbon based molecular architectures for light energy conversion, electrochemistry of fullerenes as applicable for functional devices, fullerenes as self-assembled morphological materials, semiconductor carbon nanotube optics, near-filed optics of nanotubes and energy transfer at the nanoscale level, understanding the role of metal catalysts in chiral selective growth of carbon nanotubes, and finally, computational studies on the stability of fullerenes and metallofullerenes.

Like the previous two volumes, the two-volume set of the present Handbook has been written as a hands-on reference guide for scientists working in the fields of biology and medicine, chemistry, physics, materials science, solid-state physics and nanotechnology. The coverage of topics presented is both in-depth and comprehensive. When combined with the perspectives for future research given by the contributing authors, this series makes an invaluable reference source that should be considered essential reading for both students and advanced researchers in the field. 


\section{xii Preface}

The editors would like to acknowledge the contributors for their timely submission of the state-of-the-art reviews. Last, but not least, the staff of World Scientific Publishing Co. is acknowledged for their immense help in bringing out this publication in a timely manner.

Francis D'Souza
Denton, TX, USA

Karl M. Kadish

Houston, TX, USA

February 16, 2012 


\section{List of Contributors}

\section{Junko Aimi}

National Institute for Materials

Science (NIMS)

Tsukuba 305-0047, Japan

Chapter 5 (Vol. 4)

\section{Takeshi Akasaka}

University of Tsukuba

Ibaraki 305-8577, Japan

Chapter 10 (Vol. 4)

\section{Tarek Baati}

UMR CNRS 8612 and LETIAM, EA 4041

University of Paris Sud, France

Chapter 2 (Vol. 3)

Myriam Barrejón

Universidad de Castilla-La Mancha

45071, Toledo, Spain

Chapter 6 (Vol. 4)

\section{Ajoy Kumar Bauri}

Shiga University of Medical Science

Otsu 520-2192, Japan

Current address: Bhabha Atomic

Research Center

Mumbai 400085, India

Chapter 6 (Vol. 3)
Marco Carini

University of Trieste

34127 Trieste, Italy

Chapter 1 (Vol. 3)

\section{Wei-Hung Chiang}

Case Western Reserve University

Cleveland, Ohio, USA

Current address: Industrial

Technology Research Institute

Hsinchu, Taiwan, ROC, 300

Chapter 9 (Vol. 4)

\section{Tatiana Da Ros}

University of Trieste

34127 Trieste, Italy

daros@units.it

Chapter 1 (Vol. 3)

\section{Luka Đorđević}

University of Trieste

34127 Trieste, Italy

Chapter 1 (Vol. 3)

\section{Rockford K. Draper}

The University of Texas at Dallas Richardson, TX 75080, USA

Chapter 3 (Vol. 3) 


\section{Francis D'Souza}

University of North Texas

Denton, TX 76203-5017, USA

Francis.Dsouza@unt.edu

Chapter 5 (Vol. 3)

\section{Solon Economopoulos}

Theoretical and Physical Chemistry Institute

National Hellenic Research

Foundation

Athens 11635, Greece

Chapter 1 (Vol. 4)

Michael W. Ellis

Virginia Tech

Blacksburg, VA 24601, USA

Chapter 2 (Vol. 4)

María José Gómez-Escalonilla

Universidad de Castilla-La Mancha 45071, Toledo, Spain

Chapter 6 (Vol. 4)

\section{Taku Hasobe}

Keio University

Yokohama 223-8522, Japan

hasobe@chem.keio.ac.jp

Chapter 3 (Vol. 4)

\section{Junbo Hou}

Institute for Critical Technology and Applied Science

Virginia Tech

Blacksburg, VA 24601, USA

junbo80@vt.edu;

junbo.hou@yahoo.com

Chapter 2 (Vol. 4)

\section{Libai Huang}

University of Notre Dame

Notre Dame, IN 46556, USA

Chapter 7 (Vol. 4)

\section{Tetyana Ignatova}

Lehigh University

Bethlehem, PA 18016, USA

Chapter 8 (Vol. 4)

\section{Matteo Iurlo}

Università di Bologna

I-40126 Bologna, Italy

Chapter 4 (Vol. 4)

\section{Nikolaos Karousis}

Theoretical and Physical Chemistry Institute

National Hellenic Research Foundation

Athens 11635, Greece

Chapter 1 (Vol. 4)

\section{Jelena Kolosnjaj-Tabi}

UMR CNRS 8612 and LETIAM, EA 4041

University of Paris Sud, France

Chapter 2 (Vol. 3)

\section{Naoki Komatsu}

Shiga University of Medical Science

Otsu 520-2192, Japan

nkomatsu@belle.shiga-med.ac.jp

Chapter 6 (Vol. 3) 


\section{Todd D. Krauss}

University of Rochester

Rochester, NY 14627, USA

krauss@chem.rochester.edu

Chapter 7 (Vol. 4)

\section{Wlodzimierz Kutner}

Institute of Physical Chemistry,

Polish Academy of Sciences

01-224 Warsaw, Poland

Cardinal Stefan Wyszynski

University in Warsaw

01-815 Warsaw, Poland

wkutner@ichf.edu.pl

Chapter 5 (Vol. 3)

\section{Gaurav Lalwani}

Stony Brook University

Stony Brook, NY 11794 - 5281,

USA

gaurav.lalwani@stonybrook.edu

Chapter 4 (Vol. 3)

\section{Fernando Langa}

Universidad de Castilla-La Mancha 45071, Toledo, Spain

Fernando.Langa@uclm.es

Chapter 6 (Vol. 4)

\section{Shyi-Long Lee}

National Chung-Cheng

University

Chia-Yi 62117, Taiwan

Chapter 10 (Vol. 4)

\section{Gang Liu}

Shiga University of Medical Science

Otsu 520-2192, Japan

Chapter 6 (Vol. 3)

\section{Massimo Marcaccio}

Università di Bologna

I-40126 Bologna, Italy

Chapter 4 (Vol. 4)

\section{Carole Mikoryak}

The University of Texas at Dallas

Richardson, TX 75080, USA

Chapter 3 (Vol. 3)

\section{Fathi Moussa}

UMR CNRS 8612 and LETIAM, EA 4041

University of Paris Sud, France

fathi.moussa@u-psud.fr

Chapter 2 (Vol. 3)

\section{Shigeru Nagase}

Institute for Molecular Science

Okasaki 444-8585, Japan

Chapter 10 (Vol. 4)

\section{Takashi Nakanishi}

National Institute for Materials Science (NIMS)

Tsukuba 305-0047, Japan

nakanishi.takashi@nims.go.jp

Chapter 5 (Vol. 4)

\section{Andrei M. Nemilentsau}

Lehigh University

Bethlehem, PA 18016, USA

Chapter 8 (Vol. 4)

\section{Lisa J. Nogaj}

Gannon University

Erie, PA 16541, USA

Chapter 7 (Vol. 4) 


\section{Paul Pantano}

The University of Texas at Dallas

Richardson, TX 75080, USA

pantano@utdallas.edu

Chapter 3 (Vol. 3)

\section{Francesco Paolucci}

Università di Bologna

I-40126 Bologna, Italy

francesco.paolucci@unibo.it

Chapter 4 (Vol. 4)

\section{Xiaobin Peng}

Shiga University of Medical Science

Otsu 520-2192, Japan

Current address: South China

University of Technology

Guangzhou 510640, China

Chapter 6 (Vol. 3)

\section{A. F. M. Mustafizur Rahman}

Shiga University of Medical Science

Otsu 520-2192, Japan

Current address: University of

Dhaka

Dhaka-1000, Bangladesh

Chapter 6 (Vol. 3)

\section{Stefania Rapino}

Università di Bologna

I-40126 Bologna, Italy

Chapter 4 (Vol. 4)

\section{Slava V. Rotkin}

Lehigh University

Bethlehem, PA 18016, USA

rotkin@lehigh.edu

Chapter 8 (Vol. 4)
Manolis M. Roubelakis

Massachusetts Institute of Technology

Cambridge, MA 02139-4307, USA

Chapter 7 (Vol. 3)

\section{R. Mohan Sankaran}

Case Western Reserve University

Cleveland, Ohio, USA

mohan@case.edu

Chapter 9 (Vol. 4)

\section{Piyush Sindhu Sharma}

Institute of Physical Chemistry

Polish Academy of Sciences

01-224 Warsaw, Poland

Chapter 5 (Vol. 3)

\section{Yanfei Shen}

National Institute for Materials

Science (NIMS)

Tsukuba 305-0047, Japan

Chapter 5 (Vol. 4)

\section{Balaji Sitharaman}

Stony Brook University

Stony Brook, NY 11794 - 5281, USA

balaji.sitharaman@stonybrook.edu Chapter 4 (Vol. 3)

\section{Zdeněk Slanina}

University of Tsukuba

Ibaraki 305-8577, Japan

zdenek@ims.ac.jp

Chapter 10 (Vol. 4) 


\section{Xueliang Sun}

University of Western Ontario

London, Ontario, N6A 5B9,

Canada

Chapter 2 (Vol. 4)

Henri Szwarc

UMR CNRS 8612 and LETIAM, EA 4041

University of Paris Sud, France

Chapter 2 (Vol. 3)

Nikos Tagmatarchis

Theoretical and Physical Chemistry Institute

National Hellenic Research

Foundation

Athens 11635, Greece

tagmatar@eie.gr

Chapter 1 (Vol. 4)

\section{Filip Uhlík}

Charles University in Prague

12843 Praha 2, Czech Republic

Chapter 10 (Vol. 4)

\section{Giovanni Valenti}

Università di Bologna

I-40126 Bologna, Italy

Chapter 4 (Vol. 4)

\section{María Vizuete}

Universidad de Castilla-La Mancha 45071, Toledo, Spain

Chapter 6 (Vol. 4)

\section{Georgios C. Vougioukalakis}

University of Athens

Athens 15771, Greece

Institute of Physical Chemistry,

NCSR "Demokritos"

Athens 15310, Greece

vougiok@chem.uoa.gr

Chapter 7 (Vol. 3)

\section{Feng Wang}

Shiga University of Medical Science

Otsu 520-2192, Japan

Current address: Wuhan Institute of Technology

Wuhan, China

Chapter 6 (Vol. 3)

\section{Jiajun Wang}

University of Western Ontario

London, Ontario, N6A 5B9,

Canada

Chapter 2 (Vol. 4)

\section{Ruhung Wang}

The University of Texas at Dallas

Richardson, TX 75080, USA

Chapter 3 (Vol. 3) 
This page intentionally left blank 


\section{Contents of Volumes 3 and 4}

\section{Volume 3 Medicinal and Bio-related Applications}

1. Fullerenes in Biology and Medicine

Marco Carini, Luka Đordević and Tatiana Da Ros

2. Toxicity Studies of [60]Fullerene and Carbon Nanotubes: State of the Art

Jelena Kolosnjaj-Tabi, Tarek Baati, Henri Szwarc and Fathi Moussa

3. Electrophoretic Methods to Quantify Carbon Nanotubes in Biological Cells

Paul Pantano, Rockford K. Draper, Carole Mikoryak and Rubung Wang

4. Carbon Nanotechnology in Regenerative Medicine Gaurav Lalwani and Balaji Sitharaman

5. Carbon Nanotube-Based Chemo- and Biosensors Piyush Sindhu Sharma, Francis D'Souza and Wlodzimierz Kutner

6. Separation of Left- and Right-Handed Structures of Single-Walled Carbon Nanotubes through Molecular Recognition Gang Liu, Feng Wang, Xiaobin Peng, A. F. M. Mustafizur Rabman, Ajoy Kumar Bauri and Naoki Komatsu

7. Surgery at the Molecular Level: Synthesis and Modifications of Open-Cage Fullerene Derivatives Manolis M. Roubelakis and Georgios C. Vougioukalakis 


\section{Volume 4 Materials and Fundamental Applications}

1. Functionalization of Graphene

Nikolaos Karousis, Solon Economopoulos and Nikos Tagmatarchis

2. Advanced Carbon Materials for Electrochemical Energy Conversion and Storage Junbo Hou, Jiajun Wang, Xueliang Sun and Michael W. Ellis

3. Carbon Nanomaterial-Based Molecular Architectures for Light Energy Conversion Taku Hasobe

4. Electrochemistry of Carbon Nanostructures: From Pristine Materials to Functional Devices Matteo Iurlo, Stefania Rapino, Giovanni Valenti, Massimo Marcaccio and Francesco Paolucci

5. Fullerene-Based Self-Assembled Morphological Materials Yanfei Shen, Junko Aimi and Takashi Nakanishi

6. Hybrids Involving Fullerenes and Carbon Nanotubes María Vizuete, Myriam Barrejón, María José Gómez-Escalonilla and Fernando Langa

7. Semiconductor Carbon Nanotube Optics Lisa J. Nogaj, Libai Huang and Todd D. Krauss

8. Near-Field Optics of SWNTs and FRET in their Nanoscale Complexes Tetyana Ignatova, Andrei M. Nemilentsau and Slava V. Rotkin

9. Understanding the Role of the Metal Catalyst in Carbon Nanotube Growth: Towards Chiral-Selective Growth of Single-Walled Carbon Nanotubes Wei-Hung Chiang and R. Mohan Sankaran

10. Stability Computations for Fullerenes and Metallofullerenes Zdeněk Slanina, Filip Uhlik, Shyi-Long Lee, Takeshi Akasaka and Shigeru Nagase 